\title{
Kinderbetreuung und Fertilität in Deutschland
}

\author{
Karsten Hank ${ }^{a}$, Michaela Kreyenfeld ${ }^{b}$ und C. Katharina Spieß ${ }^{c}$
}

$\underline{\text { Januar } 2004}$

Zusammenfassung: Eine zentrale Rolle in der Diskussion um eine bessere Vereinbarkeit von Familie und Beruf spielt die Versorgung mit bedarfsgerechter Kinderbetreuung. Eine umfassendes Angebot an Betreuungsplätzen fördert jedoch nicht nur die Müttererwerbstätigkeit, sondern könnte sich auch positiv auf Fertilitätsentscheidungen auswirken. Im vorliegenden Beitrag untersuchen wir auf Basis von Daten des Soziooekonomischen Panels (SOEP) und der amtlichen Kinder- und Jugendhilfestatistik den Einfluss der regionalen Verfügbarkeit von Kinderbetreuung auf das Geburtenverhalten west- und ostdeutscher Frauen in den Jahren 1996 bis 2000. Zentrales Ergebnis unserer Analyse ist, dass in den östlichen Bundesländern die Verfügbarkeit institutioneller Kinderbetreuung den Übergang zum ersten Kind positiv beeinflusst, während sich in den westlichen Bundesländern allein die Verfügbarkeit informeller Betreuung durch Großmütter als statistisch signifikant erweist. Verantwortlich hierfür dürfte in erster Linie das unterschiedliche Niveau der öffentlichen Betreuungsinfrastruktur in Ost und West sein, was sich insbesondere bei der Versorgung im Krippen- und Hortbereich sowie bei der Verfügbarkeit von Ganztagsplätzen zeigt.

a Mannheimer Forschungsinstitut Ökonomie und demographischer Wandel (MEA). Korrespondenzanschrift: MEA; Universität Mannheim; L 13, 17; 68131 Mannheim. Email: hank@mea.uni-mannheim.de.

${ }^{\mathrm{b}}$ Max-Planck-Institut für demografische Forschung. Korrespondenzanschrift: Max-Planck-Institut für demografische Forschung; Konrad-Zuse-Str. 1; 18057 Rostock. Email: kreyenfeld@demogr.mpg.de.

${ }^{c}$ DIW Berlin und Technische Universität Berlin. Korrespondenzanschrift: DIW Berlin; KöniginLuise-Str. 5; 14195 Berlin.Email: kspiess@diw.de.

Wir danken zwei anonymen Gutachtern und den Herausgebern für konstruktive Hinweise zur Überarbeitung des Manuskriptes. 


\section{Einleitung}

Die Förderung von Familien und insbesondere die Frage, wie sich Kinder und weibliche Berufskarrieren besser miteinander vereinbaren lassen, sind in Deutschland Thema eines intensiven gesellschaftspolitischen Diskurses. Eine zentrale Rolle in dieser Debatte spielt die Versorgung mit bedarfsgerechter Kinderbetreuung. Strukturelle Mängel im derzeitigen System öffentlicher bzw. öffentlich finanzierter Kinderbetreuung ermöglichen es zumindest westdeutschen Frauen oft nicht, gleichzeitig Mutter und erwerbstätig zu sein. Insbesondere das Fehlen von Betreuungsmöglichkeiten für Kinder im Krippen- und Hortalter, sowie die mangelnde Flexibilität der Öffnungszeiten von Kindergärten, gelten als wesentliche Ursachen für die mangelnde Kompatibilität von Beruf und Familie in den alten Bundesländern (z.B. Beckmannn / Kurtz 2001; Kreyenfeld et al. 2001, 2002).

Im Allgemeinen geht man davon aus, dass ein umfassendes Angebot an Betreuungsplätzen die Erwerbstätigkeit von Frauen fördert. Empirische Untersuchen aus dem anglo-amerikanischen Raum bestätigen einen Zusammenhang zwischen den Kosten sowie der Verfügbarkeit von Kinderbetreuung und dem Erwerbsverhalten von Müttern (z.B. Anderson / Levine 2000; Hofferth / Collins 2000; Stolzenberg / Waite 1984). Die wenigen deutschen Studien zu diesem Thema kommen zu unterschiedlichen Ergebnissen: Während Kreyenfeld und Hank (2000) keinen Effekt der Verfügbarkeit von Kinderbetreuungsplätzen auf das Erwerbsverhalten westdeutscher Frauen nachweisen können, zeigen differenziertere Analysen von Büchel und Spieß (2002a, 2002b) einen positiven Einfluss von Ganztagsbetreuungsmöglichkeiten auf die Wahrscheinlichkeit, dass eine westdeutsche Mutter erwerbstätig ist (vgl. auch Spieß / Büchel 2003).

In international vergleichenden Studien wird außerdem darauf hingewiesen, dass das Kinderbetreuungssystem eines Landes nicht nur für die Förderung der Frauen- bzw. 
Müttererwerbstätigkeit relevant ist, sondern dass auch ein positive Korrelation mit dem allgemeinen Fertilitätsniveau angenommen werden kann (z.B. McDonald 2000; Rindfuss / Brewster 1996; Strohmeier 2003). Empirische Studien zur Rolle der Kinderbetreuung für das individuelle Geburtenverhalten sind jedoch selten und liefern darüber hinaus wenig eindeutige Ergebnisse (vgl. Abschnitt 2).

Im vorliegenden Beitrag analysieren wir die Bedeutung der regionalen Verfügbarkeit von Kinderbetreuungsplätzen für den Übergang zum ersten Kind in West- und Ostdeutschland in den Jahren 1996 bis 2000. ${ }^{1}$ Dabei werden verschiedene Dimensionen der Kinderbetreuung berücksichtigt, nämlich das allgemeine Versorgungsniveau mit Plätzen in Tageseinrichtungen für Kinder unterschiedlicher Altersgruppen, die Art des Angebots (Halbtags- oder Ganztagsplätze), sowie der Zugang zu informeller Betreuung durch soziale Netzwerke. Damit gehen wir in zweifacher Hinsicht über eine frühere Arbeit von Hank und Kreyenfeld (2003) zum selben Thema hinaus. Während diese ihre Untersuchung auf Westdeutschland und auf die Verfügbarkeit von Kindergartenplätzen beschränken mussten, können wir nicht nur erstmals auch ostdeutsche Kreisdaten zur Kinderbetreuung in eine Fertilitätsanalyse aufnehmen, sondern können außerdem differenziertere Indikatoren der Betreuungsinfrastruktur in unsere Modelle einbeziehen.

In Abschnitt 2 werden zunächst in allgemeiner Form die notwendigen gesellschaftlichen Rahmenbedingungen für die Vereinbarkeit von Familie und Beruf diskutiert. Hierbei stehen demografische Implikationen sowie die zentrale Bedeutung der Kinderbetreuung mit ihren unterschiedlichen Dimensionen im Vordergrund. In Abschnitt 3

\footnotetext{
${ }^{1}$ Die regionale Betreuungsinfrastruktur wird hier als Teil der lokalen Opportunitätsstruktur verstanden, die zusammen mit sozialen Interaktionsprozessen und kulturellen Milieus auf räumlicher Ebene den sozialen Kontext bildet, in dem die individuelle Entscheidung für oder gegen eine Familiengründung stattfindet. Eine ausführliche theoretische und empirische Diskussion dieser Zusammenhänge findet sich bei Hank (2003).
} 
wird diese Diskussion in den bundesdeutschen Kontext der 1990er Jahre eingebunden. Die Datenbasis der empirischen Analyse und das methodische Vorgehen werden in Abschnitt 4 beschrieben, gefolgt von den multivariaten Regressionsergebnissen in Abschnitt 5. Die Resultate werden mit Blick auf ihre familienpolitische und demografische Bedeutung in Abschnitt 6 abschließend zusammengefasst und diskutiert.

\section{Müttererwerbstätigkeit, Fertilität und Kinderbetreuung im gesellschaftlichen Kontext}

Seit etwa Mitte der 1960er Jahre können in den meisten westlichen Ländern sehr niedrige Geburtenraten bei gleichzeitig zunehmendem Bildungsniveau und einer wachsenden Erwerbsorientierung von Frauen beobachtet werden. Dieses Phänomen wird vielfach mit den hohen Opportunitätskosten eines durch die Familiengründung erzwungenen Rückzugs der Frau aus dem Arbeitsmarkt erklärt (z.B. Becker 1993; Lesthaeghe 1995). ${ }^{2}$ Entsprechend argumentieren Brewster und Rindfuss (2000), dass die inverse Beziehung zwischen Fertilität und Frauenerwerbstätigkeit die Unvereinbarkeit von Kind und Beruf in modernen Industriegesellschaften widerspiegelt.

Unter bestimmten gesellschaftlichen Rahmenbedingungen ist es für Frauen jedoch durchaus möglich, Berufskarriere und Mutterschaft miteinander zu vereinbaren, was u.U. sogar zu einer Umkehrung der bislang bekannten negativen Korrelation zwischen Frauenerwerbsbeteiligung und allgemeiner Geburtenziffer führen kann (z.B. Ahn / Mira 2002; Engelhardt / Prskawetz 2004; Kögel 2004). Notwendige Voraussetzungen hierfür sind gleichberechtigte Geschlechterrollen, flexible Arbeitsbedingungen und insbesondere

\footnotetext{
${ }^{2}$ Eine weitergehende Diskussion ökonomischer und/oder soziologischer Theorien der Fertilität übersteigt den Rahmen dieses Beitrags bei weitem. Für einen aktuellen Überblick verweisen wir auf die Arbeiten von Braun (2000) und Huinink (2000).
} 
eine ausreichende Versorgung mit Möglichkeiten zur außerhäuslichen Kinderbetreuung (vgl. Ellingsæter / Rønsen 1996; McDonald 2000).

Je mehr die traditionelle Arbeitsteilung zwischen Mann und Frau abgelöst wird und Anreize geschaffen werden, dass auch Väter Betreuungsaufgaben übernehmen (z.B. Aldous et al. 1998; Sundström / Duvander 2002), desto schwächer sollte die negative Korrelation zwischen Fertilität und der Arbeitsmarktbeteiligung von Frauen ausfallen. Eine große Rolle spielen dabei neben der sozialen Akzeptanz einer Erwerbstätigkeit von Müttern (z.B. Knudsen / Wærness 2001) vor allem steuer- und familienpolitische Maßnahmen, die entweder den Fortbestand eines 'male-breadwinner' Modells fördern oder aber auf die Einbindung von Frauen in den Arbeitsmarkt zielen (z.B. Dingeldey 2002; Sainsbury 1999). Darüber hinaus spielt die Familienkompatibilität von Arbeitsplätzen eine wichtige Rolle, die z.B. durch flexible Arbeitszeiten und flexible Wiedereinstiegsregelungen für Eltern gestärkt werden könnte (vgl. Glass / Estes 1997). Als wichtigste strukturelle Voraussetzung für die Lösung des Vereinbarkeitsproblems gilt jedoch eine bedarfsgerechte Versorgung mit Kinderbetreuung (z.B. Meyers et al. 1999; Rindfuss / Brewster 1996).

Während eine relativ umfangreiche, meist US-amerikanische, Literatur zeigt, dass die Verfügbarkeit von Betreuungseinrichtungen für Kinder tatsächlich zu einer größeren Erwerbsbeteiligung von Müttern führt (vgl. für einen Überblick Merkle 1994; Spieß / Büchel 2003), ist bisher nur selten explizit untersucht worden, inwieweit sich ein ähnlicher Effekt auch für Fertilitätsentscheidungen nachweisen lässt. Die wenigen europäischen Studien zu diesem Thema erlauben, soweit sie miteinander vergleichbar sind, bislang keine systematischen Schlussfolgerungen. Während Kravdal (1996) in Norwegen und Del Boca (2002) in Italien einen schwach positiven Einfluss des Vorhandenseins von Kinderbetreuungsmöglichkeiten auf die Wahrscheinlichkeit der Geburt eines (weiteren) 
Kindes feststellen, finden sich weder in den schwedischen Daten von Andersson et al. (2004), noch bei Hank und Kreyenfeld (2003), die den Zusammenhang zwischen der Verfügbarkeit von Kindergartenplätzen und dem Geburtenverhalten westdeutscher Frauen analysieren, Hinweise auf einen solchen Effekt der Betreuungsinfrastruktur.

Dass es nur wenige fundierte Mikroanalysen zum Zusammenhang zwischen Kinderbetreuungsangeboten und Fertilitätsentscheidungen gibt, dürfte u.a. an den hohen Anforderungen an die Daten für eine solche Untersuchung liegen. Da in erster Linie die Infrastruktur des näheren Wohnumfeldes relevant ist, bedarf es eines Datensatzes, der neben der individuellen Fertilitätsbiographie auch Informationen zur kleinräumigen Versorgung mit Kinderbetreuung beinhaltet. Darüber hinaus ist es notwendig, die Betreuungsangebote in ihrer Mehrdimensionalität zu erfassen (siehe Abbildung 1; vgl. auch Andersson et al. 2004). Im Wesentlichen können drei grundlegende Betreuungsarten unterschieden werden: die elterliche Betreuung, die informelle Betreuung durch soziale Netzwerke und die institutionelle Betreuung. Die elterliche Betreuung wird durch den Vater und, hauptsächlich, die Mutter geleistet. Die Betreuung in sozialen Netzwerken umfasst die Betreuung durch Nachbarn, Freunde oder Verwandte (insbesondere die Großeltern; vgl. Vandell et al. 2003). Als institutionelle Kinderbetreuung wird die Betreuung in (öffentlich oder privat finanzierten) Tageseinrichtungen bezeichnet, wobei wir Krippen, Kindergärten und Horte unterscheiden. Schließlich kann noch eine Reihe anderer Betreuungsformen genannt werden, deren quantitative Bedeutung jedoch vergleichsweise gering ist. Hierzu zählen z.B. die Tagespflege oder die Betreuung durch Au-pairs.

Um die umfassende Betreuung eines Kindes sicherzustellen, bedarf es häufig einer Kombination dieser Betreuungsarten (z.B. Smith 2000; Tietze / Roßbach 1991), die sich alle hinsichtlich ihrer regionalen Verfügbarkeit, ihrer zeitlichen Flexibilität, ihrer Kosten, 
ihrer Qualität und ihrer sozialen Akzeptanz unterscheiden. Der folgende Abschnitt diskutiert dies ausführlicher mit Bezug auf die konkrete Situation der Bundesrepublik in den 1990er Jahren.

\section{[Abbildung 1 etwa hier einfügen]}

\section{Kinderbetreuung im vereinten Deutschland}

Ähnlich wie in anderen europäischen Ländern stellen in Deutschland öffentliche bzw. öffentlich geförderte Tageseinrichtungen die wichtigste Form außerhäuslicher Kinderbetreuung dar, während der Tagespflege oder privat-gewerblichen Betreuungseinrichtungen nur eine untergeordnete Rolle zukommt (vgl. OECD 2001; Spieß 1998). Die von den privaten Haushalten zu tragenden Kosten der Inanspruchnahme institutioneller Kinderbetreuung sind vergleichsweise niedrig. In Nordrhein-Westfalen betragen z.B. die monatlichen Elternbeiträge für Kinder aus Haushalten in mittlerer Einkommenslage (d.h. mit einem Jahreseinkommen zwischen 25.000 und $35.000 €$ ) für den Krippenbesuch ca. $140 €$, für den ganztägigen Kindergarten ca. $70 €$ und für die Nachmittagsbetreuung im Hort ca. $60 €$ (vgl. § 17 (3), Gesetz über Tageseinrichtungen für Kinder 2002; verfügbar unter http://www.tageseinrichtungen.nrw.de). Eine Analyse auf Basis des Sozio-oekonomischen Panels aus dem Jahr 1996 zeigt, dass Eltern in Deutschland im Durchschnitt drei Prozent des Haushaltsnettoeinkommens für Elternbeiträge aufwenden (Kreyenfeld et al. 2001), was deutlich unter der entsprechenden finanziellen Belastung US-amerikanischer Haushalte liegt (z.B. Smith 2000).

Kindertageseinrichtungen in Deutschland werden weitgehend über das kommunale Budget gefördert (z.B. Kreyenfeld et al. 2002). Die Versorgung mit Betreuungsplätzen hängt dabei nicht nur von den vorhandenen Finanzmitteln, sondern auch von der 
politischen Prioritätensetzung in den jeweiligen Kommunen ab. Dies führt zu einer z.T. erheblichen regionalen Variation der Versorgungsdichte im Krippen-, Kindergarten- und Hortbereich. Besonders deutliche, historisch gewachsene, Unterschiede lassen sich vor allem zwischen den neuen und den alten Bundesländern feststellen.

\section{Regionale Unterschiede zwischen Ost- und Westdeutschland}

Während die Versorgungsunterschiede bei Halbtags-Kindergartenplätzen in Ost und West noch relativ gering ausfallen, sind im Krippen- und Hortbereich erhebliche Differenzen zwischen den beiden Landesteilen zu erkennen (siehe Tabelle 1; vgl. auch Hank et al. 2001; Kreyenfeld 2003a). In Westdeutschland lag die Versorgungsquote im Hortbereich 1998 bei nur 6 Prozent, in Ostdeutschland jedoch bei fast 70 Prozent. Ein ähnliches Bild ergibt sich für den Krippenbereich, wo die ostdeutsche Versorgungsquote trotz stetigen Kapazitätsabbaus - im Jahr 1998 mit 36 Prozent immer noch mehr als 10 mal so hoch war wie in Westdeutschland. Darüber hinaus zeigt sich, dass in den westlichen Bundesländern nur ein Fünftel aller Kindergartenplätze ganztägig verfügbar sind, während dies auf fast alle Plätze in Ostdeutschland zutrifft. Eine Hauptursache für dieses Betreuungsgefälle zwischen Ost und West sind die divergierenden Ziele, mit denen in der ehemaligen DDR und der alten Bundesrepublik Kindertageseinrichtungen gefördert wurden, und z.T. hieraus resultierende Unterschiede in der sozialen Akzeptanz von außerhäuslicher Betreuung und Müttererwerbstätigkeit.

[Tabelle 1 etwa hier einfügen]

In Westdeutschland führten in den 1960er Jahren pädagogische Bemühungen um die Chancengleichheit von Kindern zu einer Expansion des staatlichen Engagements im 
Kindergartenbereich (z.B. Kreyenfeld et al. 2001). Vor diesem Hintergrund setzte sich der Halbtagskindergarten als Regelinstitution für fast alle Vorschulkinder durch. Der seit 1996 bestehende Rechtsanspruch auf einen Kindergartenplatz bezieht sich in der Regel jedoch nur auf eine vormittägliche Betreuung von vier Stunden, was die Vereinbarkeit von weiblicher Berufskarriere und Familie kaum verbessert, da unter solchen zeitlichen Restriktionen selbst eine qualifizierte Teilzeiterwerbsarbeit praktisch unmöglich ist (vgl. Büchel / Spieß 2002b). Darüber hinaus sind Schulhorte oder Ganztagsschulen in Westdeutschland bis heute eine Ausnahme (z.B. Gottschall / Hagemann 2002) und Kinderkrippen eine gesellschaftlich immer noch kaum akzeptierte Alternative zur elterlichen Betreuung. So stimmten im Jahr 2000 zwei Drittel der 30- bis 40-jährigen westdeutschen Befragten des ALLBUS der Aussage zu, „ein Kleinkind wird sicherlich darunter leiden, wenn seine Mutter berufstätig ist“ (vgl. Kreyenfeld 2003b). Hierin dokumentieren sich grundlegende subjektive Zweifel von Teilen der Bevölkerung an der Qualität außerhäuslicher Betreuung (z.B. Blau / Hagy 1998; Spieß / Tietze 2002) sowie an der Wünschbarkeit berufstätiger Mütter.

In Ostdeutschland wurde hingegen zu DDR-Zeiten ein flächendeckendes System an Betreuungsplätzen für Kinder aller Altersklassen aufgebaut, das ausdrücklich die Frauenerwerbstätigkeit fördern sollte (z.B. Trappe 1995). Die Kindertageseinrichtungen waren in der Regel ganztägig geöffnet. Entgegen früherer Erwartungen konnte bislang nicht nur ein relativ umfassende Angebot mit Ganztagsplätzen in Krippe, Kindergarten und Hort aufrechterhalten werden, sondern auch die Erwerbsneigung ostdeutscher Frauen blieb nach der Wiedervereinigung hoch. Entsprechend zeigt sich in den oben zitierten ALLBUSDaten eine deutlich geringere Zustimmung (33 \%) zu der Aussage, dass eine Erwerbstätigkeit von Müttern schädlich für Kleinkinder sei. Es ist jedoch fraglich, inwieweit die umfassenden institutionellen Betreuungsangebote in den neuen 
Bundesländern - insbesondere vor dem Hintergrund der Finanzschwäche ostdeutscher Kommunen - zukünftig bestand haben werden. Bereits in den 1990er Jahren wurde die Anzahl der Plätze in Kindertageseinrichtungen erheblich reduziert, was sich nur aufgrund des noch deutlicheren Geburtenrückgangs kaum negativ auf die Versorgungsquoten ausgewirkt hat (Hank et al. 2001; Sternitzky / Putzing 1996).

\section{Kleinräumige regionale Unterschiede auf der Kreisebene}

Neben den Unterschieden zwischen West- und Ostdeutschland zeigt sich - aufgrund der kommunalen Zuständigkeiten - auch auf kleinräumiger Ebene eine z.T. erhebliche Variation in den Betreuungsangeboten. Die kleinste regionale Einheit, für die Informationen über Betreuungsangebote in Kindertageseinrichtungen für die gesamte Bundesrepublik zur Verfügung stehen, sind die Stadt- und Landkreise.

Auf Grundlage einer eigenen Zusammenstellung von Daten aus dem Jahr 1998 veranschaulicht Abbildung 2 die Verteilung der Versorgungsquoten in den west- und ostdeutschen Kreisen. ${ }^{3}$ Hierbei zeigt sich für Ostdeutschland auf einem insgesamt deutlich höheren Niveau eine stärkere regionale Heterogenität in der Versorgung mit Betreuungsplätzen als in Westdeutschland. Während die ostdeutschen Versorgungsquoten

\footnotetext{
${ }^{3} \mathrm{Zu}$ den in Tabelle 1 ausgewiesenen Versorgungsquoten des Deutschen Jugendinstituts ergeben sich auf Grund unterschiedlicher Abgrenzungen der Altersklassen geringfügige Abweichungen (vgl. DJI, 2002: 35). Außerdem schließen die Daten des DJI Schulhorte ein, über die uns leider keine Informationen vorliegen. Dies bedeutet, dass wir die Versorgung im Hortbereich in Ostdeutschland tendenziell unterschätzen. So liegt in etwa 30 Prozent der ostdeutschen Kreise die Versorgungsquote bei Horten unter 10 Prozent, was wahrscheinlich darauf zurückzuführen ist, dass in diesen Kommunen die Betreuung von Kindern im Schulalter in erster Linie über Schulhorte erfolgt, die in unserer Statistik nicht erscheinen. - Da es heute nicht mehr möglich ist, getrennte Versorgungsquoten für Berlin-Ost und Berlin-West zu berechnen, wird in den folgenden Analysen Berlin zu Westdeutschland gezählt.
} 
im Krippenbereich etwa zwischen 10 und 65 Prozent und bei Horten im Kern zwischen 25 und 75 Prozent variieren, liegt die Versorgung mit Krippen und Horten in fast allen westdeutschen Kreisen bei unter 10 Prozent. Ein höheres Angebot findet sich lediglich in Hamburg und Berlin. In Westdeutschland gibt es allein im Kindergartenbereich eine größere und dem Osten vergleichbare Variation. Hinsichtlich der Verteilung von Ganztagsplätzen lassen sich zwischen den westdeutschen Kreisen regionale Unterschiede im Bereich unterhalb der 50-Prozent-Versorgungsquote feststellen, während die Variation in Ostdeutschland allgemein geringer ausfällt und ausschließlich im oberen Versorgungsbereich (über 80 Prozent) stattfindet, da die meisten Betreuungsplätze ganztägig verfügbar sind.

Zusammenfassend bleibt festzuhalten, dass in Westdeutschland insbesondere regionale Unterschiede der Versorgungsquoten im Kindergartenbereich und im Anteil der Ganztagsplätze beobachtet werden können, während in Ostdeutschland die Verfügbarkeit von Krippen-, Kindergarten- und Hortplätzen regional erheblich streut.

[Abbildung 2 etwa hier einfügen]

\section{Soziale Netzwerke und andere Betreuungsformen}

Angesichts des niedrigen westdeutschen Versorgungsniveaus im Krippen-, Hort- und Ganztagsbereich liegt es nahe, dass Eltern in den alten Bundesländern auf zusätzliche Betreuungsarrangements angewiesen sind. Bemerkenswert ist hierbei, dass weniger als 5 Prozent aller Eltern bezahlte Betreuungspersonen (wie die Tagespflege) als zusätzliche Betreuungsform nutzen (siehe Tabelle 2). Selbst für erwerbstätige Mütter spielt die Tagespflege mit weniger als 10 Prozent nur eine untergeordnete Rolle im 
Betreuungsarrangement (vgl. Engelbrech / Jungkunst 1998; Hank / Kreyenfeld 2003: Tabelle 2).

Eine zentrale Rolle nehmen hingegen soziale Netzwerke ein. Ein Drittel aller Kinder in Deutschland wird zumindest teilweise durch Verwandte betreut (vgl. Tabelle 2). Dass dieser Anteil in Ostdeutschland ebenso hoch ist wie in den alten Bundesländern, dürfte u.a. dadurch zu erklären sein, dass auch hier Betreuungslücken (z.B. während der Ferien) überbrückt werden müssen, oder Kinder auf dem Weg zu oder von der Tageseinrichtung begleitet werden müssen. Eine herausragende Rolle kommt dabei den Großeltern zu (z.B. Büchel / Spieß 2002b; Engelbrech / Jungkunst 1998).

[Tabelle 2 etwa hier einfügen]

\section{Datenbasis, Variablen und methodisches Vorgehen}

Datenbasis unserer Untersuchung, die sich auf den Zeitraum 1996 bis 2000 bezieht, ist das Sozio-oekonomische Panel (SOEP) (vgl. SOEP-Gruppe 2001). Das SOEP ist eine seit 1984 in Westdeutschland und seit 1990 in Ostdeutschland jährlich durchgeführte Längsschnittuntersuchung von inzwischen über 20.000 Personen. Den Individualdaten des SOEP können unter strengen Datenschutzauflagen Informationen über den Stadt- bzw. Landkreis des jeweils aktuellen Wohnorts zugespielt werden.

Die Analyse bezieht sich auf die Determinanten des Übergangs zum ersten Kind für Frauen im Alter von 18 bis 45 Jahren, wobei für West- und Ostdeutschland separate zeitdiskrete logistische Mehrebenenmodelle geschätzt werden (vgl. Barber et al. 2000; 
siehe auch Hank 2003). ${ }^{4}$ Bei dem von uns gewählten Verfahren kann die Regressionskonstante mit dem jeweiligen Kontext (hier: Kreis) variieren, d.h. sie besteht aus einer fixen und einer zufälligen Komponente ('random intercept model'). Neben zeitabhängigen und zeitunabhängigen Individualvariablen sowie zeitkonstanten Kontextvariablen findet sich auf der rechten Seite der Regressionsgleichung ein normalverteilter regionaler Zufallskoeffizient $\left(\sigma_{u}\right)$. Er beschreibt die Abweichung jedes einzelnen Kontexts vom fixen Teil der Regressionskonstante. Damit wird nicht nur für die Korrelation zwischen Individuen innerhalb desselben Kontexts kontrolliert, sondern es werden auch sonst unbeobachtete regionale Effekte erfasst. Alle Modelle wurden unter Verwendung der Funktion 'xtlogit’ mit STATA 7.0 geschätzt.

Als zeitveränderliche sozio-demografische Kontrollvariablen verwenden wir das Alter der Frau, ihren höchsten (beruflichen) Ausbildungsabschluss, sowie die Information darüber, ob sie mit einem Partner zusammenlebt. Zusätzlich gehen als zeitkonstante Individualmerkmale die Nationalität (nur für Westdeutschland) und die Zugehörigkeit zu einer Religionsgemeinschaft (gemessen im Jahr 1997) in die Regression ein. Darüber hinaus enthält das SOEP auch Informationen zum Einkommen und zum Erwerbsstatus. Da wir jedoch zeitdiskrete Modelle schätzen, denen nur jahresgenaue Informationen zu Grunde liegen, ist es nicht möglich, die zeitliche Ordnung von Erwerbstätigkeit vor Geburt und Rückzug aus dem Arbeitsmarkt aufgrund von Schwangerschaft bzw. Geburt hinreichend genau zu identifizieren. Aus diesem Grund verwenden wir das Bildungsniveau als Indikator für die Erwerbsorientierung und das Einkommenspotenzial der Frau (vgl. hierzu auch Brauns et al. 1999; Konietzka 1999).

\footnotetext{
${ }^{4}$ Synonym zum Begriff des statistischen Mehrebenenmodells werden in der Literatur auch die Bezeichnungen ‘random coefficient model' und 'hierarchical (linear) model’ verwandt. Vgl. Engel (1998) und Snijders / Bosker (1999) für ausführliche Einführungen in die Mehrebenenanalyse.
} 
Als unabhängige Kontextvariablen verwenden wir neben einem binären Stadt-LandIndikator (,kreisfreie Stadt' vs. ,Landkreis’) zunächst folgende metrischen regionalen Informationen aus dem Jahr 1998: (1) die Versorgungsquoten für den Krippen-, Kindergarten und Hortbereich, und (2) den Anteil der Ganztagsplätze an allen Plätzen. Diese Indikatoren wurden von uns auf Grundlage von Daten der Kinder- und Jugendhilfestatistik 1998 sowie der 'Statistik regional' Datenbank (Statistische Ämter der Länder und des Bundes 2000) berechnet. Da die Kinder- und Jugendhilfe bundesweit nur alle vier Jahre erhoben wird, ist es nicht möglich, Versorgungsquoten für jedes Jahr unseres Beobachtungszeitraums (1996 bis 2000) zu berechnen. Wir können jedoch davon ausgehen, dass zumindest die relativen Unterschiede in der Versorgung mit Kindertageseinrichtungen zwischen den Kreisen während der in Frage stehenden fünf Jahre stabil geblieben sind, ${ }^{5}$ die entsprechenden Variablen werden deshalb als zeitkonstante Kovariaten behandelt. Schließlich berücksichtigen wir außerdem die Möglichkeit der Kinderbetreuung durch Personen aus dem familiären Netzwerk der Befragten. Hierfür haben wir eine (zeitkonstante) binäre Variable aus den Informationen der 1996er Welle des SOEP generiert, die den Wert 1 annimmt, wenn die Mutter der Frau (d.h. die potenzielle Großmutter) im selben Ort $^{6}$ wohnt. $^{7}$

\footnotetext{
${ }^{5}$ Wegen der ostdeutschen Kreisreform Mitte der 1990er Jahre ist ein Vergleich der Daten des Jahres 1998 mit den regionalen Informationen aus der Kinder- und Jugendhilfestatistik 1994 ebenso wenig möglich wie eine Berücksichtigung weiter zurück liegender Wellen des SOEP.

${ }^{6}$ Bei der Konstruktion der Netzwerkvariable folgen wir den Antwortvorgaben des SOEP. Eine Befragte wird hier darum gebeten, anzugeben, ob die eigene Mutter noch lebt. Falls sie noch lebt, sind folgende Antwortvorgaben möglich „A: im gleichen Haus“, „B: in der Nachbarschaft“, „C: im gleichen Ort, aber weiter als 15 Minuten Fussweg entfernt“, „D: in einem anderen Ort, aber innerhalb 1 Stunde erreichbar“, „E: weiter entfernt (Deutschland)“, „F: im Ausland“. Wir haben diese Antwortvorgaben dichotomisiert in „Großmutter im Ort“ (Antwortvorgaben A-C) und „Großmusster nicht im Ort (D-F).
} 
Unser Datensatz umfasst 1.519 in Westdeutschland lebende Frauen aus 296 Kreisen sowie 545 Ostdeutsche in 101 Kreisen. Da jede Befragte mehrfach beobachtet werden kann, resultiert dies in 4.963 Beobachtungen und 263 Ereignissen für West-, sowie 1.644 Beobachtungen und 69 Ereignisse für Ostdeutschland (siehe Tabelle 3 für weitere deskriptive Statistiken).

[Tabelle 3 etwa hier einfügen]

\section{Ergebnisse der multivariaten Analysen}

Die wichtigsten Ergebnisse der multivariaten Analysen sind in Tabelle 4 dargestellt und werden im Folgenden ausführlicher diskutiert.

Kontrollvariablen. Zwischen dem Alter der Frau und der Geburt des ersten Kindes ergibt sich die erwartete glockenförmige Beziehung. Für in Westdeutschland lebende Frauen mit beruflichem Ausbildungs- oder Hochschulabschluss reduziert sich die Übergangsrate zum ersten Kind im Vergleich zu Frauen ohne Berufsabschluss um etwa 50 Prozent. Analog zu anderen Studien finden wir hier ebenfalls einen deutlichen „Institutioneneffekt“ der Bildung, d.h. die Wahrscheinlichkeit einer Mutterschaft ist am geringsten, wenn die Befragte sich noch in Ausbildung befindet (vgl. Blossfeld / Huinink 1991). In Ostdeutschland zeigt sich ein ähnlicher Zusammenhang, jedoch sind hier die Effekte der Bildungsvariablen nicht statistisch signifikant. Die Zugehörigkeit zu einer Religionsgemeinschaft hat im Osten keinen und im Westen nur einen schwach

\footnotetext{
${ }^{7}$ Ein potentielles Betreuungsnetzwerk mag ein Grund ist, warum Personen weniger mobil sind. Mit anderen Worten, die Antizipation der Familiengründung mag dazu führen, dass eine Person in der Nähe der elterlichen Haushalts wohnen bleibt. In unseren Analysen sind wir davon ausgegangen, dass derartige Überlegungen von untergeordneter Bedeutung sind und Migrationsentscheidungen in erster Linie von anderen Faktoren abhängen.
} 
signifikanten positiven Einfluss auf den Übergang zum ersten Kind. Ferner weisen in Westdeutschland lebende Ausländerinnen eine höhere Wahrscheinlichkeit auf, im Beobachtungszeitraum Mutter zu werden, als deutsche Befragte. Frauen, die mit einem Partner zusammenleben, haben erwartungsgemäß eine mehrfach höhere Erstgeburtswahrscheinlichkeit als allein lebende Frauen. Der Stadt-Land-Indikator zeigt für Ostdeutschland eine deutlich niedrigere Übergangsrate zum ersten Kind, wenn die Frau in einer kreisfreien Stadt lebt. In Westdeutschland ist dieser Zusammenhang deutlich schwächer und statistisch nicht signifikant (vgl. hierzu ausführlicher Hank 2003).

Kinderbetreuungsvariablen. In den östlichen Bundesländern beschleunigt eine hohe Versorgung mit Plätzen im Kindergarten- und Hortbereich deutlich die Geburt eines ersten Kindes. Zwar sind auch die Verfügbarkeit von Krippen- und Ganztagsplätzen positiv mit dem Übergang zur Elternschaft korreliert, der jeweilige Effekt ist jedoch statistisch nicht signifikant. In Westdeutschland übt weder die quantitative Versorgung, noch die Art des Angebots (d.h. der Anteil an Ganztagsbetreuungsplätzen) einen signifikanten Effekt auf die Fertilitätsentscheidung der Frau aus. Im Gegensatz zu Ostdeutschland erweist sich hier jedoch der Koeffizient der Variablen für eine mögliche Betreuung durch die Großmutter als positiv und signifikant (auf dem 10-Prozent-Niveau). Wohnt die Mutter der Befragten im selben Ort, erhöht sich die Übergangsrate zum ersten Kind ceteris paribus um etwa 30 Prozent (vgl. auch Hank / Kreyenfeld 2003).

[Tabelle 4 etwa hier einfügen]

In den in Tabelle 4 dargestellten Modellen wurden die Versorgungsquoten als metrische Variablen abgebildet. Um die Koeffizienten besser interpretieren zu können, haben wir in einem nächsten Schritt die gleichen Modelle mit kategorialen Kinderbetreuungsvariablen geschätzt. Dieser Modellspezifikation entspricht auch die 
Hypothese, dass zwischen institutioneller Kinderbetreuung und den Fertilitätsentscheidungen keine lineare Beziehung besteht, sondern dass von bestimmten Schwellenwerten auszugehen ist, die erreicht werden müssen, bevor die Versorgung mit Kinderbetreuung verhaltensrelevant wird und das individuelle Geburtenverhalten beeinflussen kann (z.B. Kravdal 1996).

Für die vorliegende Analyse haben wir verschiedene Schwellenwerte basierend auf der tatsächlichen Verteilung der Versorgungsquoten und der Verfügbarkeit von Ganztagsplätzen abgeleitet. Getrennt für Ost- und Westdeutschland werden unterschiedliche Werte für die Bereiche Krippe, Kindergarten und Hort verwendet, wobei für Westdeutschland der Schwellenwert jeweils bei der Hälfte der Verteilung angesetzt wird. Da die Streuung der Betreuungsquoten in allgemein in Ostdeutschland größer ist, wurde hier die jeweilige Verteilung gedrittelt und entsprechend zwei Schwellenwerte konstruiert. Die Schwellenwerte und die zugehörigen Regressionsergebnisse sind in Tabelle 5 zusammengefasst; da sich die Koeffizienten der Kontrollvariablen nicht von jenen in Tabelle 4 unterscheiden, werden sie nicht nochmals aufgeführt.

Auch bei der Verwendung kategorialer Variablen für die Versorgung mit Kinderbetreuung zeigen sich für Westdeutschland keine statistisch signifikanten Effekte. Für Ostdeutschland gilt ebenfalls der bereits oben gezeigte Zusammenhang: Je besser die Versorgung mit Kindergarten- und Hortplätzen, desto höher die Übergangsrate zum ersten Kind. Für den Hortbereich gilt, dass sich ab einer Versorgungsquote von über 60 Prozent die Übergangsrate im Vergleich zu den anderen Kategorien verdoppelt. Ein hoher Anteil an Ganztagsplätzen (über 80 Prozent) wirkt gleichfalls positiv auf die Übergangsrate zum ersten Kind, allerdings ist der Effekt hier nicht statistisch signifikant.

[Tabelle 5 etwa hier einfügen] 
In einem letzten Schritt wird eine mögliche Korrelation zwischen den verschiedenen Versorgungsquoten berücksichtigt. Besteht eine enge Beziehung zwischen den einzelnen Kontrollvariablen, können sich die einzelnen Effekte überlagern. Daher haben wir eine Modellspezifikation getestet, bei der nur für jeweils eine der drei Betreuungsformen kontrolliert wird. Die Ergebnisse sind in Tabelle 6 wiedergegeben. Es ergeben sich keine wesentlichen Abweichungen zu den vorhergehenden Befunden: ${ }^{8}$ in Ostdeutschland zeigt sich ein positiver - allerdings nicht durchgehend signifikanter Zusammenhang - zwischen einer hohen Versorgung mit institutioneller Kinderbetreuung und dem Übergang zum ersten Kind; in Westdeutschland lässt sich ein solcher Zusammenhang nicht feststellen, dafür gibt es aber Hinweise darauf, dass die Verfügbarkeit eines familialen Netzwerks sich positiv auf das Geburtenverhalten auswirkt. Bereits die Untersuchung von Hank und Kreyenfeld (2003: Tabelle A2) hat gezeigt, dass Interaktionen, etwa zwischen den Bildungsmerkmalen der Frau und den Betreuungsvariablen, zu keinen zusätzlichen Erkenntnissen führen.

Schließlich haben wir ebenfalls Analysen für den Übergang zum zweiten Kind durchgeführt. Dabei haben sich jedoch weder für West-, noch für Ostdeutschland stabile und statistisch signifikante Effekte der Verfügbarkeit institutioneller oder informeller Betreuungsarrangements gezeigt. Wir interpretieren dieses Ergebnis dahingehend, dass in ganz Deutschland mit Blick auf die Berufskarriere der Frau die Entscheidung für oder gegen ein erstes Kind die wesentliche Weichenstellung darstellt (vgl. Huinink 2002).

8 Es sei auch darauf hingewiesen, dass die verschiedenen Spezifikationen der Kinderbetreuungsvariablen nicht zu signifikanten Unterschieden hinsichtlich der Modellgüte führen. Mit einem Pseudo- $\mathrm{R}^{2}$ zwischen 0.11 und 0.14 findet sich überall eine mittlere ModellAnpassung. 
[Tabelle 6 etwa hier einfügen]

\section{Zusammenfassung und Fazit}

Ausgangspunkt der vorliegenden Untersuchung war die Hypothese, dass sich Frauen in Regionen mit einem höheren Versorgungsniveau bei Kindertageseinrichtungen und Ganztagsbetreuungsplätzen eher für eine Elternschaft entscheiden, als Frauen in Regionen mit einer schlechteren Betreuungsinfrastruktur, d.h. mit schlechteren Voraussetzungen für die Vereinbarkeit von Kind und Beruf. ${ }^{9}$ Auf der Grundlage von Daten des SOEP für die Jahre 1996 bis 2000 und regionalen Informationen zur institutionellen Betreuungssituation auf der Ebene von Stadt- und Landkreisen wurden zeitdiskrete logistische Mehrebenenmodelle für den Übergang zum ersten Kind in West- und Ostdeutschland geschätzt. Als wesentlichstes Ergebnis zeigt sich, dass in den östlichen Bundesländern eine hohe Versorgung mit Plätzen im Kindergarten- und Hortbereich den Übergang zum ersten Kind signifikant positiv beeinflusst, während dies in den westlichen Bundesländern allein für die Verfügbarkeit informeller Betreuungsformen zutrifft (vgl. auch Hank / Kreyenfeld 2003).

Die wichtigsten Gründe für diesen Befund dürften, soweit es den Westen betrifft, im insgesamt zu niedrigen Niveau der Versorgung mit Krippen-, Hort-, und Ganztagsplätzen,

\footnotetext{
${ }^{9}$ Leider konnten wir mit unseren Daten nicht untersuchen, wie die objektive Versorgung mit Kinderbetreuungsmöglichkeiten und die Chancen zur Lösung des Vereinbarkeitsproblems subjektiv wahrgenommen werden. Möglicherweise treffen Frauen ihre Entscheidungen auf der Grundlage falscher oder unvollständiger Informationen. So könnte z.B. insbesondere in Ostdeutschland die heutige Betreuungssituation schlechter eingeschätzt werden, als sie tatsächlich ist. Gleichzeitig schließen niedrige Versorgungsquoten in einem bestimmten Stadt- oder Landkreis natürlich nicht aus, dass es auch dort individuell durchaus möglich sein kann, Erwerbs- und Erziehungsarbeit miteinander zu verknüpfen.
} 
sowie in der z.T. sehr geringen regionalen Variation liegen. Das Fehlen von Ganztagsbetreuungsmöglichkeiten im Kindergartenbereich dürfte Hauptursache für die mangelnden Signifikanz der Versorgungsquote für die 3- bis 6-jährigen Kinder sein. In einer solchen Situation erscheint es plausibel, dass Frauen (bzw. Paare) bei der Familienplanung den Parameter 'institutionelle Kinderbetreuung' unabhängig vom jeweiligen Wohnort als irrelevant betrachten, denn sie müssen davon ausgehen, dass das öffentlich finanzierte Kinderbetreuungssystem in den alten Bundesländern insgesamt keine ausreichenden Rahmenbedingungen für die Vereinbarkeit von Familie und Erwerbstätigkeit bietet. Es überrascht daher nicht, dass sich das soziale, bzw. familiäre Netzwerk als bedeutsam erweist. Die Nähe einer 'Großmutter' hat in Westdeutschland einen signifikant positiven Einfluss auf die Geburt des ersten Kindes. Die zunehmende Erwerbsbeteiligung der Großmüttergeneration und eine mögliche Verlängerung der Lebensarbeitszeit könnte jedoch dazu führen, dass dieser Pool potenzieller Betreuungspersonen zukünftig nur noch in geringerem Ausmaß zur Verfügung steht (vgl. Büchel / Spieß 2002b).

In Ostdeutschland, wo eine deutlichere regionale Variation der Versorgungsquoten auf einem insgesamt hohen Niveau vorliegt, zeigt sich eine positive Korrelation zwischen der Verfügbarkeit institutioneller Kinderbetreuung und dem Übergang zum ersten Kind. Bei der Versorgung mit Krippenplätzen erweist sich der entsprechende Koeffizient jedoch nicht als statistisch signifikant. Dies könnte darauf zurückzuführen sein, dass trotz der hohen Erwerbsorientierung von Frauen in den neuen Bundesländern auch hier die Elternzeit häufig in Anspruch genommen wird (z.B. Engelbrech / Jungkunst 1998). Das relativ gut ausgebaute Angebot an Ganztagsbetreuungsmöglichkeiten erlaubt danach jedoch einen relativ problemlosen Wiedereinstieg von Müttern in den Arbeitsmarkt. In den ostdeutschen Bundesländern war also in den 1990er Jahren die Vereinbarkeit von Kind und 
Beruf weitgehend gegeben. Dies hat sich nicht nur in der hohen Erwerbsbeteiligung von Müttern widergespiegelt, sondern ostdeutsche Frauen bekamen darüber hinaus ihr erstes Kind immer noch in einem jüngeren Alter als Westdeutsche (vgl. Kreyenfeld 2003b). Aufgrund des Rückgangs der periodenspezifischen Geburtenziffern ist dieser Aspekt meist übersehen worden. Es ist zu befürchten, dass die ostdeutschen Kommunen nicht in der Lage sein werden, die bislang gute Betreuungsinfrastruktur auch zukünftig zu erhalten, was sich langfristig negativ auf die Fertilitätsentwicklung in den neuen Bundesländern auswirken könnte.

Würde ein Ausbau der Kinderbetreuungsinfrastruktur einen positiven Effekt auf das Geburtenverhalten westdeutscher Frauen haben? In diesem Artikel haben wir uns auf den Übergang zum ersten Kind konzentriert, der u.E. angesichts des hohen Alters bei Erstgeburt und des hohen Anteils kinderloser Frauen in Westdeutschland das 'kritische' biografische Ereignis darstellt (z.B. Dorbritz 2001). Unsere empirischen Ergebnisse deuten darauf hin, dass nur geringfügige Änderungen im westdeutschen Betreuungssystem keinen Effekt auf das Familiengründungsverhalten haben würden. Vielmehr bedürfte es einer durch parallele Änderungen in anderen familienpolitisch relevanten Bereichen (z.B. dem Einkommensteuersystem) flankierten, deutlichen Ausweitung des Angebots an Krippenplätzen und Ganztagsbetreuungsmöglichkeiten, um erwerbsorientierten Frauen zu signalisieren, dass Kind und Beruf miteinander vereinbar sind - und dass dies auch gesellschaftlich gewünscht wird. 


\section{Literatur}

Ahn, N. / Mira, P. (2002): A note on the changing relationship between fertility and female employment rates in developed countries. Journal of Population Economics 15: 667682.

Aldous, J. / Mulligan, G.M. / Bjarnason, T., 1998: Fathering over Time: What Makes the Difference? Journal of Marriage and the Family 60: 809-820.

Anderson, P.M. / Levine, P.B., 2000: Child Care and Mothers’ Employment Decisions. S. 420-462 in: D. Card / R. Blank (Hrsg.), Finding Jobs: Work and Welfare Reform. New York: Russel Sage.

Andersson, G. / Duvander, A.-Z. / Hank, K., 2004: Do Child Care Characteristics Influence Continued Childbearing in Sweden? An Analysis of the Quantity, Quality, and Price Dimension. Journal of European Social Policy 14: im Druck.

Barber, J.S. / Murphy, S.A. / Axinn, W.A. / Marples, J., 2000: Discrete-time multilevel hazard analysis. S. 201-235 in: M.E. Sobel / M.P. Becker (Hrsg.), Sociological Methodology 30. Washington: Blackwell Publishers.

Becker, G.S., 1993: A Treatise on the Family (Erweiterte Auflage). Cambridge: Harvard University Press.

Beckmann, P. / Kurtz, B., 2001: Die Betreuung der Kinder ist der Schlüssel. IABKurzbericht, Heft 10: 1-7.

Blau, D.M. / Hagy, A.P., 1998: The Demand for Quality in Child Care. Journal of Political Economy 106: 104-146.

Blossfeld, H.-P. / Huinink, J., 1991: Human capital investments or norms of role transition? How women's schooling and career affect the process of family formation. American Journal of Sociology 97: 143-168. 
Braun, N., 2000: Ökonomische Theorien in der Bevölkerungswissenschaft. S. 298-338 in: U. Müller et al. (Hrsg.), Handbuch der Demographie, Bd. 1: Modelle und Methoden. Berlin u.a.: Springer.

Brauns, H. / Steinmann, S. / Kieffer, A. / Marry, C., 1999: Does education matter? France and Germany in comparative perspective. European Sociological Review 15: 61-89.

Brewster, K.L. / Rindfuss, R.R., 2000: Fertility and women’s employment in industrialized nations. Annual Review of Sociology 26: 271-296.

Büchel, F. / Spieß, C.K., 2002a: Kindertageseinrichtungen und Müttererwerbstätigkeit Neue Ergebnisse zu einem bekannten Zusammenhang. Vierteljahrshefte zur Wirtschaftsforschung 71: 96-114.

Büchel, F. / Spieß, C.K., 2002b: Form der Kinderbetreuung und Arbeitsmarktverhalten von Müttern in West- und Ostdeutschland. Schriftenreihe des Bundesministeriums für Familie, Senioren, Frauen und Jugend, Band 220. Stuttgart: Kohlhammer.

Del Boca, D., 2002: The effect of child care and part time opportunities on participation and fertility decisions in Italy. Journal of Population Economics 15: 549-573.

Deutsches Jugendinstitut (DJI), 2002: Zahlenspiegel. Tageseinrichtungen für Kinder. München: Deutsches Jugendinstitut.

Dingeldey, I., 2002: Das deutsche System der Ehegattenbesteuerung im europäischen Vergleich. WSI-Mitteilungen 3/2002: 154-160.

Dorbritz, J., 2001: Familienbildungsverläufe der Generationen 1950 und 1965 im Vergleich. BiB-Mitteilungen 1/2001: 10-14.

Engel, U., 1998: Einführung in die Mehrebenenanalyse. Opladen: Westdeutscher Verlag.

Engelbrech, G. / Jungkunst, M., 1998: Erwerbsbeteiligung von Frauen und Kinderbetreuung in ost- und westdeutschen Familien. IAB Werkstattbericht 2/98. Nürnberg: Institut für Arbeitsmarkt und Berufsforschung. 
Engelhardt, H. / Prskawetz, A., 2004: On the changing correlation between fertility and female employment over space and time. European Journal of Population 20, im Druck.

Ellingsæter, A.L. / Rønsen, M., 1996: The dual strategy: motherhood and the work contract in Scandinavia. European Journal of Population $12:$ 239-260.

Glass, J.L. / Estes, S.B., 1997: The family responsive workplace. Annual Review of Sociology 23: 289-313.

Gottschall, K. / Hagemann, K., 2002: Die Halbtagsschule in Deutschland: Ein Sonderfall in Europa? Aus Politik und Zeitgeschichte, Nr. 41-42: 12-22.

Hank, K., 2003: Eine Mehrebenenanalyse regionaler Einflüsse auf die Familiengründung westdeutscher Frauen in den Jahren 1984 bis 1999. Kölner Zeitschrift für Soziologie und Sozialpsychologie 55: 79-98.

Hank, K. / Kreyenfeld, M., 2003: A Multilevel Analysis of Child Care and Women’s Fertility Decisions in Western Germany. Journal of Marriage and Family 65: 584596.

Hank, K. / Tillmann, K. / Wagner, G.G., 2001: Außerhäusliche Kinderbetreuung in Ostdeutschland vor und nach der Wiedervereinigung. Ein Vergleich mit Westdeutschland in den Jahren $1990 \quad$ - 1999. Zeitschrift für Bevölkerungswissenschaft 26: 55-65.

Hofferth, S. / Collins, N., 2000: Child care and employment turnover. Population Research and Policy Review 19: 357-395.

Huinink, J., 2000: Soziologische Ansätze zur Bevölkerungsentwicklung. S. 339-386 in: U. Müller et al. (Hrsg.), Handbuch der Demographie, Bd. 1: Modelle und Methoden. Berlin u.a.: Springer.

Huinink, J., 2002: Polarisierung der Familienentwicklung in europäischen Ländern im Vergleich. S. 47-73 in: N. Schneider / H. Matthias-Bleck (Hrsg.), Elternschaft heute. Gesellschaftliche Rahmenbedingungen und individuelle 
Gestaltungsaufgaben. Zeitschrift für Familienforschung (Sonderheft 2). Opladen: Leske + Budrich.

Knudsen, K. / Wærness, K., 2001: National Context, Individual Characteristics and Attitudes on Mothers' Employment: A Comparative Analysis of Great Britain, Sweden and Norway. Acta Sociologica 44: 67-79.

Kögel, T., 2004: Did the association between fertility and female employment in OECD countries really change its sign? Journal of Population Economics 17: im Druck.

Konietzka, D., 1999: Die Verberuflichung von Marktchancen. Die Bedeutung des Ausbildungsberufs für die Platzierung im Arbeitsmarkt. Zeitschrift für Soziologie 28: $379-400$.

Kravdal, Ø., 1996,): How the local supply of day-care centers influences fertility in Norway: A parity-specific approach. Population Research and Policy Review 15, 201-218.

Kreyenfeld, M., 2003a: Kinderbetreuung und soziale Ungleichheit. In: R. Becker / W. Lauterbach (Hrsg.), Bildung als Privileg? Ursachen von Bildungsungleichheit aus soziologischer Sicht. Opladen: Lesek + Budrich, im Druck.

Kreyenfeld, M., 2003b: Crisis or adaptation reconsidered: A comparison of East and West German fertility patterns in the first six years after the ,Wende'. European Journal of Population 19: 303-329.

Kreyenfeld, M. / Hank, K., 2000: Does the availability of child care influence the employment of mothers? Findings from western Germany. Population Research and Policy Review 19: 317-337.

Kreyenfeld, M. / Spieß, C.K. / Wagner, G.G., 2001: Finanzierungs- und Organisationsmodelle institutioneller Kinderbetreuung. Neuwied: Luchterhand.

Kreyenfeld, M. / Spieß, C.K. / Wagner, G.G., 2002: Kinderbetreuungspolitik in Deutschland: Möglichkeiten nachfrageorientierter Steuerungs- und Finanzierungsinstrumente. Zeitschrift für Erziehungswissenschaft 5: 201-221. 
Lesthaeghe, R., 1995: The second demographic transition in Western countries: an interpretation. S. 17-62 in: K.O. Mason / A.-M. Jensen (Hrsg.), Gender and family change in industrialized countries. Oxford: Clarendon Press.

McDonald, P., 2000: Gender Equity, Social Institutions and the Future of Fertility. Journal of Population Research 17: 1-16.

Merkle, L.E., 1994: Frauenerwerbstätigkeit und Kinderbetreuung, Heidelberg: PhysicaVerlag.

Meyers, M.K. / Gornick, J.C. / Ross, K.E., 1999: Public Childcare, Parental Leave, and Employment. S. 117-146 in: D. Sainsbury (Hg.), Gender and Welfare State Regimes. Oxford: Oxford University Press.

OECD, 2001: Starting Strong. Early Childhood Education and Care Policy. Paris: OECD.

Rindfuss, R.R. / Brewster, K.L. (1996): Childrearing and fertility. Population and Development Review 22 (Supplement): 258-289.

Sainsbury, D., 1999: Taxation, Family Responsibilities, and Employment. S. 185-209 in: D. Sainsbury (Hg.), Gender and Welfare State Regimes. Oxford: Oxford University Press.

Smith, K., 2000: Who’s Minding the Kids? Child Care Arrangements: Fall 1995. Current Population Reports, P70-70. Washington, DC: U.S. Census Bureau.

Snijders, T.A.B. / R.J. Bosker, 1999: Multilevel analysis: An introduction to basic and advanced multilevel modeling. London: Sage.

SOEP-Gruppe, 2001: The German Socio-Economic Panel (GSOEP) after more than 15 years - Overview. S. 7-14 in: E. Holst et al. (Hrsg.), Proceedings of the Fourth International Conference of German Socio-Economic Panel Study Users (GSOEP2000), Vierteljahrshefte zur Wirtschaftsforschung 70.

Spieß, C.K., 1998: Staatliche Eingriffe in Märkte für Kinderbetreuung. Analysen im deutsch-amerikanischen Vergleich. Frankfurt a. M. und New York: Campus. 
Spieß, C.K. / Büchel, F., 2003: Effekte der regionalen Kindergarteninfrastruktur auf das Arbeitsangebot von Müttern. In: W. Schmähl (Hg.), Wechselwirkungen zwischen Arbeitsmarkt und sozialer Sicherung II, Reihe „Schriften des Vereins für Socialpolitik“. Berlin: Duncker \& Humblot, 95-126.

Spieß, C.K. / Büchel, F. / Frick, J.R., 2002: Kinderbetreuung in West- und Ostdeutschland: Sozioökonomischer Hintergrund entscheidend. DIW-Wochenbericht 31/02: 518-524.

Spieß, C.K. / Tietze, W., 2002: Qualitätssicherung in Kindertageseinrichtungen - Gründe, Anforderungen und Umsetzungsüberlegungen für ein Gütesiegel. Zeitschrift für Erziehungswissenschaft 5: 139-162.

Statistische Ämter der Länder und des Bundes, 2000: Statistik regional (CD-ROM), Ausgabe 2000. Wiesbaden u.a.

Sternitzky, A. / Putzing, M., 1996: Kollabiert die Kinderversorgung? - Soziale Einrichtungen im Wandel. S. 17-39 in: H. Bertram (Hg.), Regionen im Vergleich: gesellschaftlicher Wandel in Ostdeutschland am Beispiel ausgewählter Regionen. Opladen: Leske + Budrich.

Stolzenberg, R.M. / Waite, L.J., 1984: Local labor markets, children and labor force participation of wives. Demography 21: 157-170.

Strohmeier, K.-P., 2003: Familienpolitik und Familienleben in Europa. S. 109-120 in: J. Dorbritz / J. Otto (Hrsg.), Familienpolitik und Familienstrukturen. Materialien zur Bevölkerungswissenschaft, Heft 108. Wiesbaden: Bundesinstitut für Bevölkerungsforschung.

Sundström, M. / Duvander, A.-Z., 2002: Family division of childcare and the sharing of parental leave among new parents in Sweden. European Sociological Review 18: 433-447.

Tietze, W. / Roßbach, H.G., 1991: Die Betreuung von Kindern im vorschulischen Alter. Zeitschrift für Pädagogik 37: 555-579. 
Trappe, H., 1995: Emanzipation oder Zwang? Frauen in der DDR zwischen Beruf, Familie und Sozialpolitik. Berlin: Akademie Verlag.

Vandell, D.L. / McCartney, K. / Owen, M.T. / Booth, C. / Clarke-Stewart, A., 2003: Variations in Child Care by Grandparents During the First Three Years. Journal of Marriage and Family 65: 375-381. 


\section{Tabellen \& Abbildungen}

Tabelle 1: Zeitliche Entwicklung des Angebots von Kinderbetreuungsplätzen in Westund Ostdeutschland, 1990 bis 1998

\begin{tabular}{|c|c|c|c|c|c|c|}
\hline & \multicolumn{3}{|c|}{ Westdeutschland } & \multicolumn{3}{|c|}{ Ostdeutschland } \\
\hline & 1990 & 1994 & 1998 & 1990 & 1994 & 1998 \\
\hline \multicolumn{7}{|c|}{ Versorgungsquoten } \\
\hline \multicolumn{7}{|c|}{$\begin{array}{l}\text { (Plätze je } 100 \text { Kinder der } \\
\text { jeweiligen Altersgruppe) }\end{array}$} \\
\hline Krippe & 2 & 2 & 3 & 54 & 42 & 36 \\
\hline Kindergarten & 78 & 73 & 87 & 114 & 96 & 112 \\
\hline Hort & 6 & 5 & 6 & 51 & 60 & 68 \\
\hline \multicolumn{7}{|c|}{$\begin{array}{l}\text { Anteil der Ganztagsplätze } \\
\text { (an allen Bereuungsplätzen in \%) }\end{array}$} \\
\hline Krippe & -- & -- & 85 & -- & -- & 86 \\
\hline Kindergarten & -- & -- & 19 & -- & -- & 98 \\
\hline Hort & -- & -- & 79 & -- & -- & 96 \\
\hline
\end{tabular}

Quelle: DJI (2002: 34, 56).

Tabelle 2: Regelmäßige Betreuung von Kindern in West- und Ostdeutschland durch Personen außerhalb des Haushalts im Jahr 2000 (Spaltenprozente)

\begin{tabular}{|c|c|c|c|c|c|c|}
\hline & \multicolumn{3}{|c|}{ Westdeutschland } & \multicolumn{3}{|c|}{ Ostdeutschland } \\
\hline & $\begin{array}{c}\text { Krippen- } \\
\text { alter }\end{array}$ & $\begin{array}{l}\text { Kindergarten- } \\
\text { alter }\end{array}$ & $\begin{array}{c}\text { Schul- } \\
\text { alter }\end{array}$ & $\begin{array}{c}\text { Krippen- } \\
\text { alter }\end{array}$ & $\begin{array}{l}\text { Kindergarten- } \\
\text { alter }\end{array}$ & $\begin{array}{l}\text { Schul- } \\
\text { alter }\end{array}$ \\
\hline Verwandte & 32 & 36 & 23 & 31 & 36 & 30 \\
\hline Freunde & 8 & 7 & 4 & 6 & 7 & 3 \\
\hline $\begin{array}{l}\text { Bezahlte } \\
\text { Betreuung }\end{array}$ & 2 & 3 & 3 & 1 & 2 & 2 \\
\hline Fallzahlen & 507 & 1.023 & 1.557 & 121 & 218 & 436 \\
\hline
\end{tabular}

Anmerkungen:

(1) Mehrfachnennungen für jedes Kind möglich.

Quelle: SOEP 2000 in Spieß et al. (2002). 
Tabelle 3: Deskriptive Statistiken

\begin{tabular}{|c|c|c|}
\hline & Westdeutschland & Ostdeutschland \\
\hline \multicolumn{3}{|l|}{ Alter der Befragten } \\
\hline Alter $18-20$ & $12 \%$ & $19 \%$ \\
\hline Alter 21-25 & $22 \%$ & $31 \%$ \\
\hline Alter 26-30 & $27 \%$ & $25 \%$ \\
\hline Alter 31-45 & $33 \%$ & $16 \%$ \\
\hline \multicolumn{3}{|l|}{ Bildung } \\
\hline In Ausbildung & $13 \%$ & $21 \%$ \\
\hline Kein Abschluss & $20 \%$ & $15 \%$ \\
\hline Ausbildungsabschluss & $53 \%$ & $47 \%$ \\
\hline Hochschulabschluss & $14 \%$ & $18 \%$ \\
\hline Fehlende Angaben & $4 \%$ & $6 \%$ \\
\hline \multicolumn{3}{|l|}{ Nationalität } \\
\hline Deutsch & $75 \%$ & -- \\
\hline Andere & $25 \%$ & -- \\
\hline \multicolumn{3}{|l|}{ Konfessionszugehörigkeit } \\
\hline Konfessionslos & $12 \%$ & $57 \%$ \\
\hline Konfessionszugehörig & $74 \%$ & $24 \%$ \\
\hline Fehlende Angaben & $14 \%$ & $19 \%$ \\
\hline \multicolumn{3}{|l|}{ Partnerschaftsstatus } \\
\hline Partner im Haushalt & $47 \%$ & $37 \%$ \\
\hline Kein Partner im Haushalt & $53 \%$ & $63 \%$ \\
\hline \multicolumn{3}{|l|}{ Stadt-Land-Indikator } \\
\hline Landkreis & $65 \%$ & $86 \%$ \\
\hline Kreisfreie Stadt & $35 \%$ & $14 \%$ \\
\hline \multicolumn{3}{|l|}{ Soziales Netzwerk } \\
\hline "Großmutter" im Ort & $22 \%$ & $23 \%$ \\
\hline "Großmutter" nicht im Ort & $57 \%$ & $48 \%$ \\
\hline Fehlende Angaben & $21 \%$ & $29 \%$ \\
\hline \multicolumn{3}{|l|}{ Versorgungsquoten } \\
\hline Krippe & $2 \%$ & $38 \%$ \\
\hline Kindergarten & $100 \%$ & $126 \%$ \\
\hline Hort & $6 \%$ & $39 \%$ \\
\hline \multicolumn{3}{|l|}{ Angebotsart } \\
\hline Anteil Ganztagsplätze & $25 \%$ & $93 \%$ \\
\hline \multicolumn{3}{|l|}{ Fallzahlen } \\
\hline Beobachtungen & 4.963 & 1.644 \\
\hline Personen & 1519 & 545 \\
\hline Anzahl Kreise & 296 & 101 \\
\hline Ereignisse & 263 & 69 \\
\hline
\end{tabular}

Anmerkungen:

(1) Bei den Ausländerinnen handelt es sich um Befragte der SOEP-Stichprobe B, d.h. Griechinnen, Italienerinnen, Spanierinnen, Türkinnen und Frauen aus Ex-Jugoslawien, die bereits 1984 in der Bundesrepublik gelebt haben.

Quelle: SOEP 1996-2000 (eigene Berechnungen). 
Tabelle 4: Zeitdiskretes logistisches Mehrebenenmodell für den Übergang zum ersten Kind - Institutionelle Kinderbetreuung als metrische Variable

\begin{tabular}{|c|c|c|c|c|c|c|}
\hline & \multicolumn{3}{|c|}{ Westdeutschland } & \multicolumn{3}{|c|}{ Ostdeutschland } \\
\hline & b & $\mathrm{xp}(\mathrm{b})$ & $\mathrm{t}$ & $\mathrm{b}$ & $\exp (b$ & $\mathrm{t}$ \\
\hline \multicolumn{7}{|l|}{ Alter der Befragten } \\
\hline Alter 18 bis 20 & -0.92 & 0.40 & $-1.66 *$ & -0.88 & 0.42 & -1.19 \\
\hline Alter 21 bis 25 & 0.07 & 1.07 & 0.38 & -0.37 & 0.69 & -1.22 \\
\hline Alter 26 bis 30 & 0 & 1 & & 0 & 1 & \\
\hline Alter 31 bis 45 & -0.14 & 0.87 & -0.92 & -0.82 & 0.44 & $-2.07 * *$ \\
\hline \multicolumn{7}{|l|}{ Bildung } \\
\hline In Ausbildung & -1.82 & 0.16 & $-3.44 * * *$ & -0.79 & 0.46 & -1.22 \\
\hline Kein Abschluss & 0 & 1 & & 0 & 1 & \\
\hline Ausbildungsabschluss & -0.62 & 0.54 & $-3.59 * * *$ & -0.35 & 0.70 & -0.68 \\
\hline Hochschulabschluss & -0.77 & 0.46 & $-2.98 * * *$ & -0.42 & 0.66 & -0.68 \\
\hline \multicolumn{7}{|l|}{ Nationalität } \\
\hline Deutsch & 0 & 1 & & & & \\
\hline Andere & 0.27 & 1.31 & $1.72 *$ & -- & & \\
\hline \multicolumn{7}{|l|}{ Konfessionszugehörigkeit } \\
\hline Konfessionslos & -0.40 & 0.67 & $-1.84 *$ & -0.32 & 0.73 & -1.03 \\
\hline Konfessionszugehörig & 0 & 1 & & 0 & 1 & \\
\hline \multicolumn{7}{|l|}{ Partnerschaftsstatus } \\
\hline Partner im Haushalt & 2.14 & 8.48 & $10.46 * * *$ & 1.95 & 7.02 & $5.55 * * *$ \\
\hline Kein Partner im Haushalt & 0 & 1 & & 0 & 1 & \\
\hline \multicolumn{7}{|l|}{ Stadt-Land-Indikator } \\
\hline Landkreis & 0 & 1 & & 0 & 1 & \\
\hline Kreisfreie Stadt & -0.22 & 0.80 & -1.10 & -1.43 & 0.24 & $-2.30 * *$ \\
\hline \multicolumn{7}{|l|}{ Soziales Netzwerk } \\
\hline "Großmutter" im Ort & 0.28 & 1.32 & $1.81 *$ & 0.12 & 1.13 & 0.41 \\
\hline "Großmutter " nicht im Ort & 0 & 1 & & 0 & 1 & \\
\hline \multicolumn{7}{|l|}{ Versorgungsquoten } \\
\hline Krippe & -2.11 & 0.12 & -0.62 & 0.65 & 1.91 & 0.67 \\
\hline Kindergarten & 0.14 & 1.15 & 0.26 & 1.42 & 4.13 & $2.39 * *$ \\
\hline Hort & 1.42 & 4.13 & 0.55 & 1.04 & 2.82 & $1.65 *$ \\
\hline \multicolumn{7}{|l|}{ Angebotsart } \\
\hline Anteil Ganztagsplätze & -1.01 & 0.37 & -1.24 & 1.99 & 7.31 & 1.17 \\
\hline Konstante & -3.64 & 0.03 & $-5.82 * * *$ & -7.56 & 0.00 & $-3.62 * * *$ \\
\hline$\sigma_{u}$ & 0.001 & & & 0.001 & & \\
\hline Log likelihood & \multicolumn{3}{|c|}{-934} & \multicolumn{3}{|c|}{-284} \\
\hline Pseudo R ${ }^{2}$ & \multicolumn{3}{|c|}{0.14} & \multicolumn{3}{|c|}{0.13} \\
\hline
\end{tabular}

Anmerkungen:

(1) Signifikanz: $*<.10 * *<.05 ; * * *<.01$.

(2) Für fehlende Angaben zu Bildungsstatus, sozialem Netzwerk und Konfessionszugehörigkeit wurde durch Einbeziehung entsprechender Indikatorvariablen in die Regression kontrolliert.

(3) Die Versorgungsquoten und der Anteil an Ganztagsbetreuungsplätzen wurden durch 100 dividiert.

Quelle: SOEP 1996-2000 (eigene Berechnungen). 
Tabelle 5: Zeitdiskretes logistisches Mehrebenenmodell für den Übergang zum ersten Kind - Institutionelle Kinderbetreuung als kategoriale Variable

\begin{tabular}{|c|c|c|c|c|c|c|}
\hline \multirow{4}{*}{\begin{tabular}{|l|} 
\\
Versorgungsquote Krippe \\
0 bis unter $5 \%$
\end{tabular}} & \multicolumn{3}{|c|}{ Westdeutschland } & \multicolumn{3}{|c|}{ Ostdeutschland } \\
\hline & \multirow[t]{2}{*}{$\mathrm{b}$} & $\exp (b)$ & \multirow[t]{2}{*}{$\mathrm{t}$} & \multicolumn{2}{|c|}{ b $\quad \exp (\mathrm{b})$} & \multirow[t]{2}{*}{$\mathrm{t}$} \\
\hline & & & & & & \\
\hline & -0.20 & 0.81 & -1.15 & 0 & 1 & \\
\hline 5 bis unter $20 \%$ & 0 & 1 & & 0 & 1 & \\
\hline 20 bis unter $50 \%$ & 0 & 1 & & 0.48 & 1.61 & 0.62 \\
\hline Über 50\% & 0 & 1 & & 0.48 & 1.61 & 0.59 \\
\hline \multicolumn{7}{|c|}{ Versorgungsquote Kindergarten } \\
\hline 0 bis unter $100 \%$ & -0.09 & 0.91 & -0.66 & 0 & 1 & \\
\hline 100 bis unter $130 \%$ & 0 & 1 & & 0 & 1 & \\
\hline 130 bis unter $140 \%$ & 0 & 1 & & 0.47 & 1.61 & 1.38 \\
\hline Über 140\% & 0 & 1 & & 0.76 & 2.13 & $2.00 * *$ \\
\hline \multicolumn{7}{|c|}{ Versorgungsquote Hort } \\
\hline 0 bis unter $5 \%$ & -0.09 & 0.91 & -0.47 & 0 & 1 & \\
\hline 5 bis unter $20 \%$ & 0 & 1 & & 0 & 1 & \\
\hline 20 bis unter $60 \%$ & 0 & 1 & & 0.19 & 1.21 & 0.49 \\
\hline Über 60\% & 0 & 1 & & 0.77 & 2.15 & $1.83 *$ \\
\hline \multicolumn{7}{|c|}{ Anteil Ganztagsbetreuung } \\
\hline 0 bis unter $20 \%$ & 0.08 & 1.08 & 0.44 & 0 & 1 & \\
\hline 20 bis unter $80 \%$ & 0 & 1 & & 0 & 1 & \\
\hline 80 bis unter $90 \%$ & 0 & 1 & & 0.59 & 1.80 & 0.73 \\
\hline Über 90\% & 0 & 1 & & 0.83 & 2.29 & 1.04 \\
\hline Log likelihood & \multicolumn{3}{|c|}{-962} & \multicolumn{3}{|c|}{-281} \\
\hline Pseudo $\mathrm{R}^{2}$ & \multicolumn{3}{|c|}{0.11} & \multicolumn{3}{|c|}{0.14} \\
\hline
\end{tabular}

Anmerkungen:

(1) Signifikanz: $*<.10 * *<.05 ; * * *<.01$.

(2) Weitere Kovariaten sind Alter, Bildung, Konfessionszugehörigkeit, Partnerschaftsstatus, sowie der Stadt-Land-Indikator. Außerdem wurde für unbeobachtete regionale Heterogenität sowie für fehlende Angaben zu Bildungsstatus, sozialem Netzwerk und Konfessionszugehörigkeit kontrolliert.

(3) Versorgungsquoten von über 100\% sind nicht in erster Linie auf Überkapazitäten zurückzuführen, sondern darauf, dass die in der Bevölkerungs- sowie der Kinderund Jugendhilfestatistik verwendeten Altersklassen nicht vollständig kompatibel sind.

Quelle: SOEP 1996-2000 (eigene Berechnungen). 
Tabelle 6: Zeitdiskretes logistisches Mehrebenenmodell für den Übergang zum ersten Kind - Separate Modelle für jede Betreuungsform

\begin{tabular}{|c|c|c|c|c|c|c|}
\hline & \multicolumn{3}{|c|}{ Westdeutschland } & \multicolumn{3}{|c|}{ Ostdeutschland } \\
\hline & $\mathrm{b}$ & $\exp (\mathrm{b})$ & $\mathrm{t}$ & $\mathrm{b}$ & $\exp (b)$ & $\mathrm{t}$ \\
\hline \multicolumn{7}{|c|}{ (a) Versorgungsquote Krippe } \\
\hline 0 bis unter $5 \%$ & -0.22 & 0.80 & -1.28 & 0 & 1 & \\
\hline 5 bis unter $20 \%$ & 0 & 1 & & 0 & 1 & \\
\hline 20 bis unter $50 \%$ & 0 & 1 & & 0.59 & 1.80 & 0.78 \\
\hline Über 50\% & -- & & & 0.63 & 1.88 & 0.81 \\
\hline Log likelihood & \multicolumn{3}{|c|}{-965} & \multicolumn{3}{|c|}{-284} \\
\hline Pseudo $\mathrm{R}^{2}$ & \multicolumn{3}{|c|}{0.11} & \multicolumn{3}{|c|}{0.13} \\
\hline \multicolumn{7}{|c|}{ (b) Versorgungsquote Kindergarten } \\
\hline 0 bis unter $100 \%$ & -0.10 & 0.90 & -0.76 & -- & & \\
\hline 100 bis unter $130 \%$ & 0 & 1 & & 0 & 1 & \\
\hline 130 bis unter $140 \%$ & 0 & 1 & & 0.65 & 1.92 & $2.00 * *$ \\
\hline Über $140 \%$ & -- & & & 0.70 & 2.01 & $2.22 * *$ \\
\hline Log likelihood & \multicolumn{3}{|c|}{-964} & \multicolumn{3}{|c|}{-283} \\
\hline Pseudo $\mathrm{R}^{2}$ & \multicolumn{3}{|c|}{0.11} & \multicolumn{3}{|c|}{0.13} \\
\hline
\end{tabular}

\section{(c) Versorgungsquote Hort}

\begin{tabular}{|c|c|c|c|c|}
\hline 0 bis unter $5 \%$ & -0.09 & $0.92-0.53$ & 0 & 1 \\
\hline 5 bis unter $20 \%$ & 0 & 1 & 0 & 1 \\
\hline 20 bis unter $60 \%$ & 0 & 1 & -0.21 & $0.81-0.65$ \\
\hline Über 60\% & -- & & 0.42 & $1.53 \quad 1.31$ \\
\hline Log likelihood & & -963 & & -284 \\
\hline Pseudo $\mathrm{R}^{2}$ & & 0.11 & & 0.13 \\
\hline
\end{tabular}

\section{(d) Anteil Ganztagsbetreuung}

\begin{tabular}{|c|c|c|c|c|c|}
\hline 0 bis unter $20 \%$ & 0.03 & $1.03 \quad 0.22$ & 0 & 1 & \\
\hline 20 bis unter $80 \%$ & 0 & 1 & 0 & 1 & \\
\hline 80 bis unter $90 \%$ & 0 & 1 & 0.71 & 2.02 & 0.91 \\
\hline Über 90\% & -- & & 0.55 & 1.74 & 0.73 \\
\hline Log likelihood & & -964 & & -283 & \\
\hline Pseudo $\mathrm{R}^{2}$ & & 0.11 & & 0.13 & \\
\hline
\end{tabular}

Anmerkungen:

(1) Signifikanz: $*<.10 * *<.05 ; * * *<.01$.

(2) Weitere Kovariaten sind Alter, Bildung, Konfessionszugehörigkeit, Partnerschaftsstatus, sowie der Stadt-Land-Indikator. Außerdem wurde für unbeobachtete regionale Heterogenität sowie für fehlende Angaben zu Bildungsstatus, sozialem Netzwerk und Konfessionszugehörigkeit kontrolliert.

(3) Versorgungsquoten von über 100\% sind nicht in erster Linie auf Überkapazitäten zurückzuführen, sondern darauf, dass die in der Bevölkerungs- sowie der Kinderund Jugendhilfestatistik verwendeten Altersklassen nicht vollständig kompatibel sind.

Quelle: SOEP 1996-2000 (eigene Berechnungen). 
Abbildung 1: Formen und Dimensionen von Kinderbetreuung

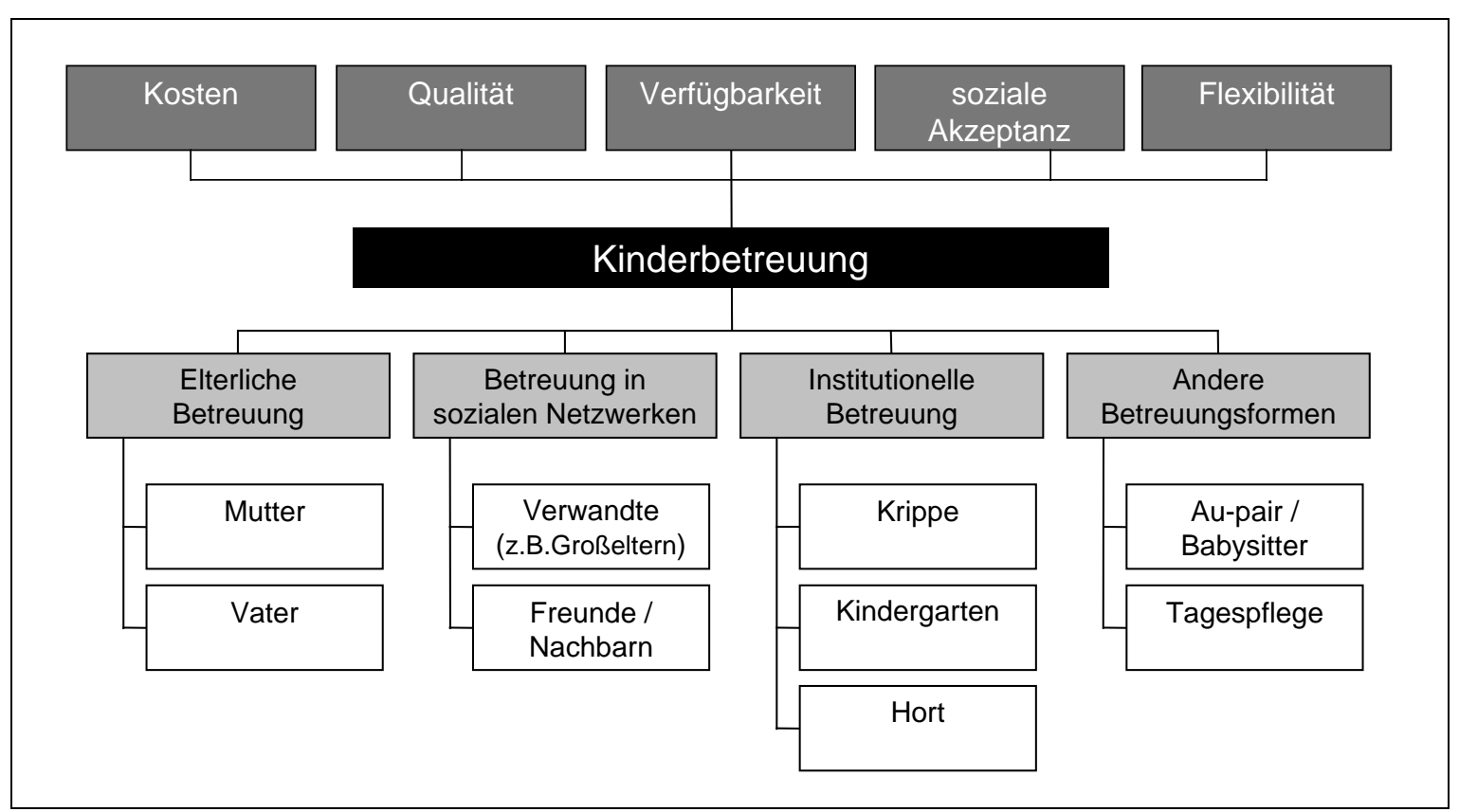

Quelle: Eigene Darstellung. 
Abbildung 2: Verteilung der Versorgungsquoten im Krippen-, Kindergarten- und Hortbereich, sowie Anteil der Ganztagsplätze in west- und ostdeutschen Stadt- und Landkreisen, 1998

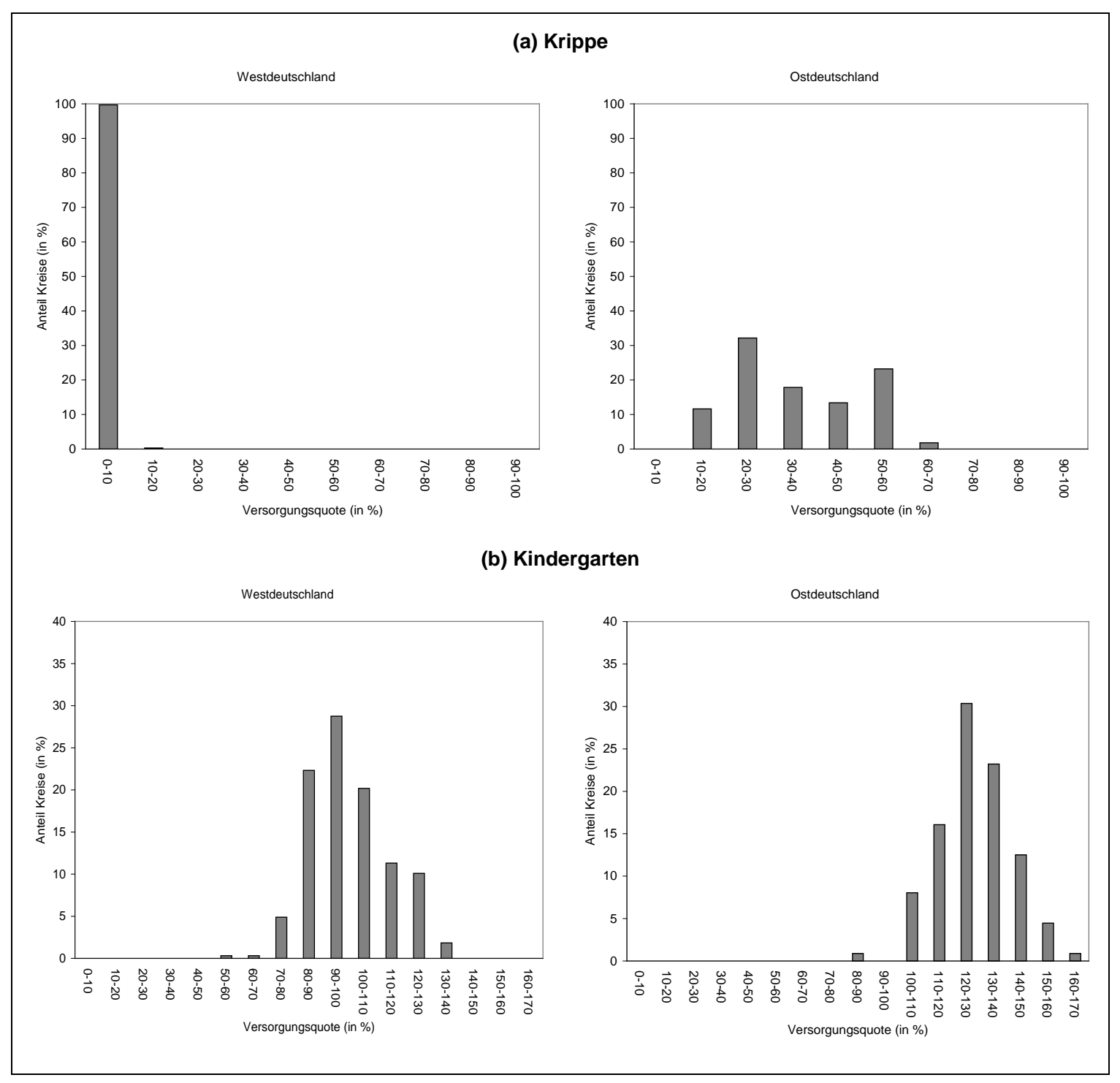

Fortsetzung nächste Seite ... 
Abbildung 2 (Fortsetzung): Verteilung der Versorgungsquoten im Krippen-, Kindergarten- und Hortbereich, sowie Anteil der Ganztagsplätze in west- und ostdeutschen Stadt- und Landkreisen, 1998

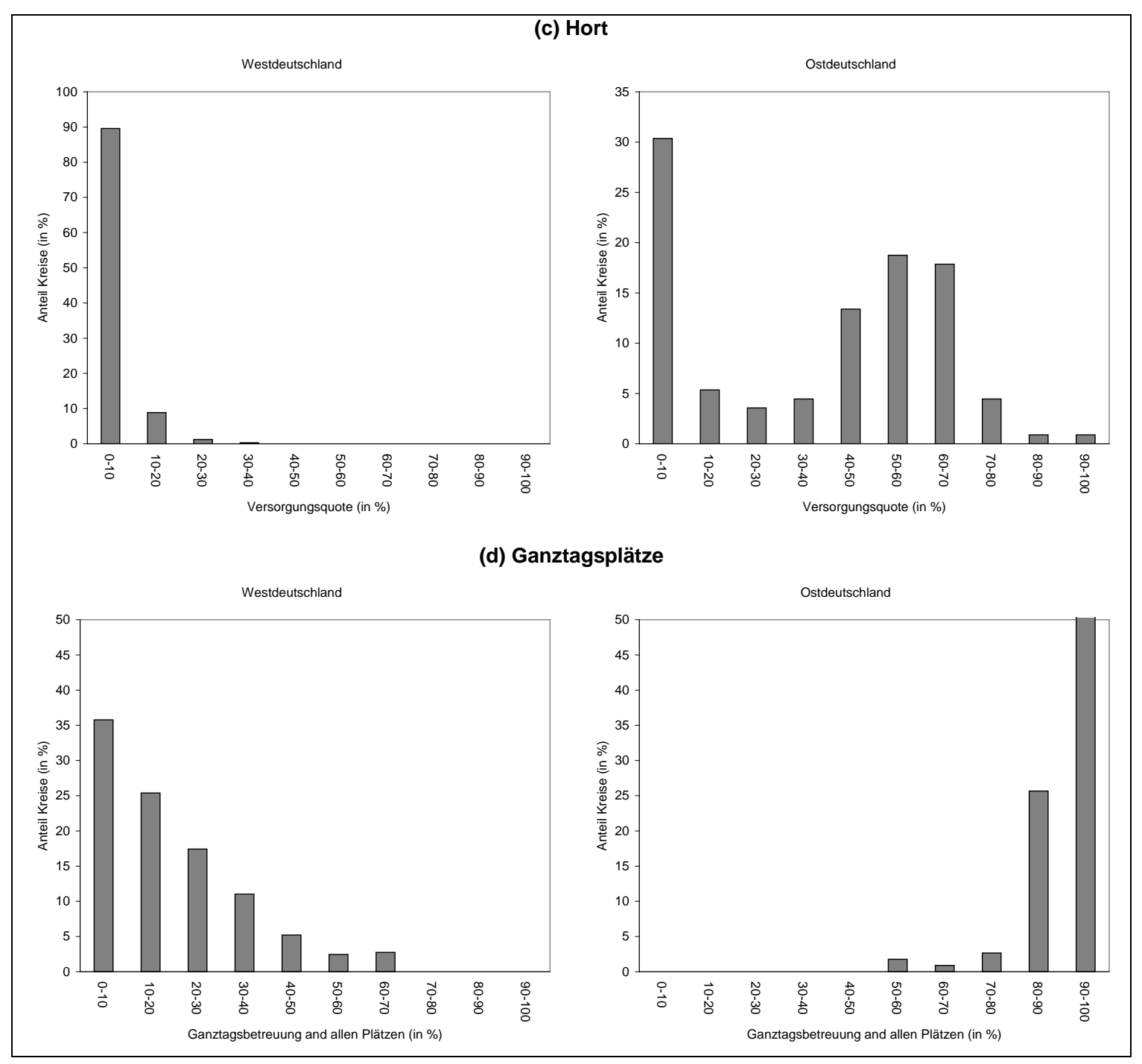

Anmerkungen:

(1) Der ehemalige Kreis Eisenach wurde wegen Zuordnungsproblemen nach der ostdeutschen Kreisreform aus der Analyse ausgeschlossen.

(2) Versorgungsquoten von über $100 \%$ sind nicht in erster Linie auf Überkapazitäten zurückzuführen, sondern darauf, dass die in der Bevölkerungs- sowie der Kinder- und Jugendhilfestatistik verwendeten Altersklassen nicht vollständig kompatibel sind.

Quelle: Eigene Zusammenstellung auf Basis der Kinder- und Jugendhilfestatistik sowie Statistik regional. 


\title{
Child Care and Fertility in Germany
}

\begin{abstract}
The provision of child care plays a crucial role in the current discussion about a better compatibility of family and employment. The availability of day-care for children may not only support the employment of mothers, but it might also have a positive effect on fertility decisions. In this paper, we use data from the German SocioEconomic Panel (SOEP) and official statistics to investigate the role of the regional supply of child care in East and West German women's childbearing behaviour in the years 1996 to 2000. Main result of our analysis is that the availability of public day-care has a positive impact on the transition to the first child in the eastern states, while in the western states only the availability of informal care by grandmothers has a statistically significant effect. The primary reason for this is likely to be the very different levels of child care provision in East and West, particularly regarding the day-care infrastructure for toddlers and school-age children and the supply of full-time care.
\end{abstract}

Karsten Hank (“Kinderbetreuung und Fertilität ...”): geb. 1971 in Recklinghausen. Studium der Sozialwissenschaft in Bochum. Promotion in Rostock. Von 1999 bis 2003 wissenschaftlicher Mitarbeiter am Max-Planck-Institut für demografische Forschung, Rostock; seit 2003 wissenschaftlicher Mitarbeiter im SHARE-Projekt am Mannheimer Forschungsinstitut Ökonomie und demographischer Wandel, Universität Mannheim. Forschungsschwerpunkte: Familiensoziologie, Demographie, empirische Sozialforschung. Wichtigste Publikationen: Regional Social Contexts and Individual Fertility Decisions, European Journal of Population 18, 2002; Sex Preferences for Children Revisited: New Evidence from Germany (mit H.-P. Kohler), Population_E 58, 2003; A Multilevel Analysis of Child Care and Women's Fertility Decisions in Western Germany (mit M. Kreyenfeld), Journal of Marriage and Family 65, 2003.

Michaela Kreyenfeld (“Kinderbetreuung und Fertilität ...”): geb. 1969 in Dortmund. Studium der Sozialwissenschaft in Bochum. 1996-1998 wissenschaftliche Mitarbeiterin am Lehrstuhl für Sozialpolitik der Ruhr-Universität Bochum. 1999-2001 Promotionsstipendiatin am Max-Planck Institut für demografische Forschung, Rostock. Seit 2002 wissenschaftliche Mitarbeiterin am Max-Planck-Institut für demografische Forschung, Rostock. 
Forschungsschwerpunkte: Familiensoziologie, Lebensverlaufsforschung, angewandte Sozialforschung. Neuere Publikationen: Women's employment and non-marital childbearing: A comparison between East and West Germany in the 1990s. Population_E 57, 2002 (mit D. Konietzka). Time-squeeze, partner effect or selfselection? An investigation into the positive effect of women's education on second birth risks in West Germany. Demographic Research 7, 2002. Crisis or adaptation reconsidered: A comparison of East and West German fertility in the first six years after the 'Wende', European Journal of Population 19, 2003. A Multilevel Analysis of Child Care and Women's Fertility Decisions in Western Germany, Journal of Marriage and Family 65, 2003 (mit K. Hank).

C. Katharina Spieß (“Kinderbetreuung und Fertilität ...”): [...] 